\title{
COMUNICACIÓN
}

\section{Estudio preliminar sobre los distintos estadios de Toxocara cati en gatos}

\author{
NATALIA CARDILLO*, ADRIANA ROSA*, e IRMA SOMMERFELT**
}

\section{PRELIMINARY STUDY ON THE DIFFERENT STAGES OF Toxocara cati IN CATS}

Toxocara cati larvae are able to migrate through the tissues of the cat, remaining infective. The objective of this study was to determine the presence of different stages of $\boldsymbol{T}$. cati in naturally infected cats. Twelve cats found dead in the street were necropsied and samples of faeces were collected for coproparasitological analysis. Samples of muscle, the lungs, kidneys, liver, mammary gland and brain were removed. They were processed by the rapid artificial digestion technique to detect $\boldsymbol{T}$. cati larvae. Coproparasitological analysis was positive for T. cati by $25 \%$, to Toxascaris leonina at 16 , $66 \%$ and $8.33 \%$ to both species. The $50 \%$ of animals presented adult parasites of $\boldsymbol{T}$. cati, of which $20 \%$ were positive to T. leonina. Statistically significant differences were found between the presence of adult parasites and cat's age. No statistically significant differences were observed in regard to sex. No larvae of $\boldsymbol{T}$. cati were recovered from tissues. The differences in the results obtained between the coproparasitological technique and the necropsy could be due to the presence of immature stages of the parasites or to intermittent elimination of eggs in faeces. The absence of larvae in tissues raises several questions related to the migratory behaviour of $\boldsymbol{T}$. cati larvae. Increasing the number of felines studied will contribute to ensure the validity of the results and will provide a better understanding about the behavior of $\boldsymbol{T}$. cati larvae during the migratory phase in the cat.

Key words: Toxocara cati, Cats parasites, Zoonosis.

\section{INTRODUCCIÓN}

Toxocara cati (mystax) es un helminto gastrointestinal que parasita frecuentemente al gato doméstico. Su gran potencial biótico y la resistencia de sus huevos en el ambiente, hacen de este una fuente de infección para hospedadores definitivos y paraténicos, dentro de los cuales se encuentra el hombre ${ }^{1,2}$.

La capacidad migratoria de las larvas de Toxocara canis y $T$. cati por los tejidos y su permanencia en estado de hipobiosis ha sido demostrada en la infección experimental en el modelo animal murino ${ }^{3-8}$.

En los gatos, luego de la ingesta de huevos larvados de $T$. cati se produce la eclosión en el intestino, las larvas atraviesan la pared y por medio de la circulación sanguínea llegan al hígado, corazón y pulmón ${ }^{9}$. Las larvas que son expectoradas y deglutidas, desarrollan en el intestino delgado los estadios adultos del parásito. Algunas larvas, continúan migrando por el organismo y se enquistan en los tejidos del gato, permaneciendo en estado infectivo.

* Cátedra de Parasitología, Facultad de Ciencias Veterinarias, Universidad de Buenos Aires. Argentina.

** Cátedra de Veterinaria en Salud Pública, Facultad de Ciencias Veterinarias,Universidad de Buenos Aires, Buenos Aires, Argentina. 
En la hembra felina, las larvas presentes en los tejidos en estado de hipobiosis se reactivan durante la preñez, y por medio de la leche se transmiten a los cachorros en el momento de la lactancia ${ }^{10}$.

En un estudio experimental se observó que la presencia de larvas en la leche se produce cuando las mismas se encuentran en la fase migratoria, hecho que ocurre cuando la gata se infesta en la última etapa de la preñez. El autor plantea que el hallazgo de larvas en tejidos periféricos obedece a migraciones erráticas del parásito ${ }^{11}$.

El objetivo del presente estudio fue determinar la presencia de distintos estadíos de $T$. cati en gatos naturalmente infectados.

\section{MATERIAL Y MÉTODO}

Selección de los animales: Se analizaron los cadáveres de 12 gatos hallados muertos en la vía pública, en la Ciudad Autónoma de Buenos Aires. Los gatos se clasificaron según sexo y edad aproximada.

Toma de muestras y análisis: Se tomaron muestras de materia fecal del recto. Se realizó el análisis coproparasitológico de las mismas por la técnica de flotación con solución azucarada saturada de Benbrook ${ }^{12}$. Se efectuó la necropsia de cada gato, con apertura y observación del aparato digestivo desde el esófago hasta el ano. En los casos en que se encontraron parásitos adultos, se clasificaron teniendo en cuenta su localización y sus características morfológicas. Se tomaron muestras $(10 \mathrm{~g})$ de los músculos cuadriceps femoral y bíceps braquial, y la totalidad de los pulmones, riñones, hígado, glándula mamaria y cerebro. Las mismas fueron analizadas por medio de la técnica de digestión artificial rápida para detectar la presencia de larvas de $T$. cati en tejidos ${ }^{13}$.

Se utilizó el software InfoStat (2006 p.1) para el procesamiento estadístico de los datos. Se realizó el test $\mathrm{T}$ student para la determinación de significación estadística entre la presencia de parasitosis y la edad del animal, y el test de proporciones por la prueba exacta de Fisher.

\section{RESULTADOS}

El análisis coproparasitológico fue positivo a T. cati en 3 gatos ( $25 \%)$, a T.s leonina en 2 gatos $(16,66 \%)$ y en uno a ambas especies (8,33\%). (Tabla 1). En el 50\% de los animales se observó la presencia de parásitos adultos de $T$. cati, de los cuales el $20 \%$ presentó parasitosis mixta con T. leonina. La existencia de parásitos adultos fue estadísticamente significativa en los gatos menores de 1 año con respecto a los adultos ( $\mathrm{p}=0,04 ; 95 \% \mathrm{IC}=24,5-108,8)$. No se encontró asociación estadística significativa entre el sexo y la presencia de parasitismo. ( $\mathrm{p}>$ $0,05)$ En el análisis de las muestras de órganos y tejidos no se detectaron larvas de $T$. cati.

\section{DISCUSIÓN}

La prevalencia observada de T. cati (25\%) por análisis coproparasitológico, es semejante a lo hallado por otros autores en Brasil $(19,1 \%)^{14}$; España $(18,3 \%)^{15}$ y Argentina $(35,7 \%)^{16}$.

Los resultados obtenidos en el diagnostico parasitológico por necropsia y por análisis de materia fecal fue observado previamente ${ }^{17}$.

La menor prevalencia de coproparasitológico en gatos, según edad y sexo

\begin{tabular}{llcll}
\hline Gato & Edad & Sexo & Necropsia & Coproparasitológico \\
\hline 1 & $<1$ año & M & T. leonina & T. leonina \\
2 & $<1$ año & M & T. cati- T. leonina & T. cati - T. leonina \\
3 & $<1$ año & M & T. cati - T. leonina & T cati \\
4 & $<1$ año & M & T. cati- T. leonina & - \\
5 & $<1$ año & H & T. cati & T cati \\
6 & $<1$ año & M & T. cati & - \\
7 & Adulto & H & T. cati & - \\
8 & Adulto & M & - & - \\
9 & Adulto & M & - & - \\
10 & Adulto & H & - & - \\
11 & Adulto & H & - & - \\
12 & Adulto & H & - & - \\
\hline
\end{tabular}
$T$. leonina con respecto a $T$. cati, coincide con lo hallado en felinos de Brasil ${ }^{14,18}$; España $^{15}$ y Alemania ${ }^{19}$. Se ha descrito la existencia de un aparente efecto inhibidor de T. cati sobre el desarrollo de T. leonina ${ }^{20}$.

La prevalencia de $T$. cati que resultó significativamente mayor en los felinos menores al año de edad y la ausencia 
de asociación estadística con respecto al sexo, son coincidentes con lo observado en gatos de Estados Unidos ${ }^{21}$; y difiere con lo hallado en gatos de Irán ${ }^{22}$, en los cuales no se observó asociación con la edad de los animales.

La ausencia de larvas de T. cati en los tejidos analizados podría deberse a lo planteado en un estudio experimental en gatos, en el cual se sugiere que la presencia de larvas en tejidos periféricos obedece a migraciones erráticas del parásito y que durante la fase migratoria no se produce enquistamiento ${ }^{11}$.

Las diferencias obtenidas en los resultados con el análisis coproparasitológico y con la necropsia podrían responder a la presencia de estadios inmaduros de T. cati y T. leonina, o a la eliminación intermitente de huevos por materia fecal. La ausencia de larvas migrantes en los tejidos de los animales positivos a T. cati plantea diversos interrogantes que condicionarían el comportamiento migratorio de las larvas en el gato, tales como la influencia de las distintas fuentes de infección, estado fisiológico del animal al momento de la misma y el efecto de reinfecciones. Los resultados de este trabajo muestran la necesidad de incrementar la cantidad de felinos estudiados y realizar estudios bajo condiciones controladas de infección. Esto contribuirá a garantizar la validez de los resultados y a un mayor conocimiento sobre el comportamiento de las larvas de $T$. cati durante la fase migratoria en los tejidos del gato.

\section{RESUMEN}

Las larvas de $T$. cati tienen la capacidad de realizar migraciones por los tejidos del gato, permaneciendo infectivas. El objetivo del presente estudio fue determinar la presencia de distintos estadíos de $T$. cati en gatos naturalmente infectados. Se efectuó la necropsia de 12 gatos hallados muertos en la vía pública. Se tomaron muestras de materia fecal para análisis coproparasitológico y muestras de músculo, la totalidad de los pulmones, riñones, hígado y cerebro. Se utilizó la técnica de digestión artificial rápida para detectar la presencia de larvas de Toxocara cati en los tejidos. El análisis coproparasitológico fue positivo a $T$. cati en un $25 \%$, a Toxascaris leonina en un $16,66 \%$ y en un $8,33 \%$ a ambas especies. El $50 \%$ de los animales presentó parásitos adultos de T. cati, de los cuales el $20 \%$ presentó parasitosis mixta con T. leonina. La existencia de parásitos adultos y su relación con la edad del animal fue estadísticamente significativa. No resultó estadísticamente significativa con respecto al sexo. En el análisis de las muestras de órganos y tejidos no se detectaron larvas de $T$. cati. Las diferencias obtenidas en los resultados con la técnica coproparasitológica y con la necropsia podrían deberse a la presencia de estadios inmaduros o a la eliminación intermitente de huevos por materia fecal. La ausencia de larvas en los tejidos plantea diversos interrogantes que podrían condicionar el comportamiento migratorio de las mismas en el gato. Es necesario incrementar la cantidad de felinos estudiados y realizar estudios bajo condiciones controladas de infección para obtener un mayor conocimiento sobre el comportamiento de las larvas de T. cati durante la fase migratoria en el gato.

\section{REFERENCIAS}

1.- PEARSONS J C. Ascarid infections of Cats and Dogs. Vet Clinics of North Am: Small Anim Pract 1987; 17: 1307-37.

2.- OVERGAAUW P A M. Aspects of Toxocara epidemiology: Toxocarosis in dogs and cats. Crit Rev Microbiol 1997; 23: 233-51.

3.- OSHIMA T. Standarization of techniques for infecting mice with Toxocara canis and observations on the normal migration routes of the larvae. J Parasitol 1961; 652-6.

4.- PROKOPIC J, FIGALlOVÁ V. Migration of some roundworm species in experimentally infected white mice. Folia Parasitológica (PRAHA) 1982; 29: 30913.

5.- ABO-SHEHADA M, AL-ZUBAIDY B, HERBERT I. The migration of larval Toxocara canis in mice. Migration Through the intestine in primary infections. Vet Parasitol 1984; 17: 65-73.

6.- COX D M, HOLLAND C V. The relationship between numbers of larvae recovered from the brain of Toxocara canis-infected mice and social beha-viour and anxiety in the host. Prasitology 1998; 116: 6, 579- 94.

7. HRCKOVA G, VELEBNÝ S, TOMASOVICOVÁ O. et al. Pathomorphological changes in mice infected with Toxocara cati following administration of febendazole and glucan. Acta Parasitológica 2001; 46: 313-20.

8.- FOK E. Pathogenesis, pathomechanism, chemotherapy and prevention of larval Toxocarosis. Thesis for Ph.D degree. Department of parasitology and Zoology. Faculty of Veterinary Medicine at Budapest. 2002.

9.- SPRENT J F. Life history and development of Toxocara cati (Schrank 1788) in the domestic cat. Parasitology 1956; 46: 54-78.

10.- SWERCZEK T W, NIELSEN S W, HELMBOLDT C F. Transmammary passage of Toxocara cati in the cat. A 
J Vet Res 1971; 32: 89-92.

11.- COATI N, SCHNIEDER T, EPE C. Vertical transmission of Toxocara cati Schrank 1788 (Anisakidae) in the cat. Parasitol Res 2004; 92: 142- 6.

12.- DOLCETTI M. Modificación al método de Benbrook mediante la centrifugación doble con solución de azúcar para el examen microscópico de las materias fecales de los animales domésticos. Rev C E Med Año 1947; XVI, No 24: 29-35.

13.- GAMBLE H R, BESSONOV A S, CUPERLOVIC K, et al. International Commission on Trichinellosis: Recommendations on methods for the control of Trichinella in domestic and wild animals intended for human consumption. Vet Parasitol 2000; 93: 393408.

14.- BARRIENTOS C M, ANTUNES C M, ALONSO R. Examen parasitológico de fezes de gatos (Felis catus domesticus) domiciliados e errantes da Região Metropolitana do Rio de Janeiro, Brasil. Rev Soc Bras Med Trop 2003; 36: 331-4.

15.- MONTOYA A, FUENTES I, JIMÉNEZ S, et al. Prevalencia de parásitos intestinales e gatos vagabundos, gatos de explotación y gatos de propietario en España. Enf Emerg 2004; 6: 211.

16.- SOMMERFELT I E, CARDILLO N, LÓPEZ C, et al.
Prevalence of Toxocara cati and other parasites in cats' faeces collected from the open spaces of public institutions: Buenos Aires, Argentina. Vet Parasitol 2006; 140: 296-301.

17.- DUBEY J P. Toxocara cati and other intestinal parasites of cats. Vet Rec 1966; 79: 506-8.

18.- MUNDIM T C D, OLIVEIRA S D, RODRIGUES D C, CURY M C. Frecuencia de helmintos em gatos de Uberlandia, Minas Gerais Arq Bras Med Vet Zootec 2004; 56(4): 562-3.

19.- EPE C, COATI N, SCHNIEDER T. Results of parasitological examinations of faecal samples from horses, ruminants, pigs, dogs, cats, hedgehogs and rabbits between 1998 and 2002. Dtsch Tierarztl Wochenschr 2004; 111: 243-7.

20.- SPRENT J F A. The life history and development of Toxascaris leonina (von Linstow 1902) in the dog and cat. Parasitology 1959; 49: 330-71.

21.- VISCO R J, CORWIN R M, SELBY L A. Effect of age and sex on the prevalence of intestinal parasitism in cats. J Am Vet Med Assoc 1978; 172(7): 797-800.

22.- SADJJADI S M, ORYANA, JALAI A R, MEHRABANI D. Prevalence and intensity of infestation with Toxocara cati in stray cats in Shiraz, Iran Veterinarski Arhiv 2001; 71: 149-57.
Correspondencia a:

Natalia Cardillo

Av. Chorroarín 280, 1427 Buenos Aires, Argentina.

E-mail:ncardillo@fvet.uba.ar 